\title{
Implications for sensor readout concepts in biomedical point of care settings with respect to robust and reliable recordings
}

\author{
$\underline{S v e n}$ Ingebrandt $^{1,2}$, Thanh C. Nguyen ${ }^{2}$, Ruben Lanche ${ }^{2}$, Jannick Wilhelm ${ }^{2}$, Dipti Rani ${ }^{1}$, Vivek \\ Pachauri $^{1}$ \\ ${ }^{1}$ Department of Computer Sciences and Microsystem Technology, University of Applied Sciences \\ Kaiserslautern, 66482 Zweibrücken, Germany \\ ${ }^{2}$ RAM Group DE GmbH, Research and Development Center, 66482 Zweibrücken, Germany \\ sven.ingebrandt@hs-kl.de
}

\begin{abstract}
:
Point of care testing of biomolecule levels in patients' blood or urine is a key future application especially for de-centralized concepts of modern healthcare. One of the discussed platform solutions where remarkable sensitivities were reported by many groups in the field are arrays of silicon nanowire sensors. The miniaturization of classical ion-sensitive field-effect transistor (ISFET) devices into state-of-the-art nanobiosensors is targeted by many researchers nowadays. The ultra-low power consumption of silicon nanowire devices compared to their much larger ISFET counterparts holds great promise for portable devices. However, besides a remarkable sensitivity resulting from the miniaturization the researchers also buy in huge cross-sensitivities to side parameters such as temperature variations, $\mathrm{pH}$ changes and ionic changes in the test solutions. This contribution addresses some of these issues and suggests possible implications for the design of robust, portable solutions for the future healthcare market.
\end{abstract}

Key words: Biomedical sensor, point of care testing, readout concept, robust recording

\section{Introduction}

In many biomedical applications, compact and portable point of care sensors are a modern demand. [1] Many of the applied sensor concepts utilize electrochemical routines for the specific identification and detection of the target analytes. In these concepts most of the time enzymes are utilized, which metabolize the target molecules. The biosensors are then designed to detect the byproducts or side parameters of the metabolic consumption such as $\mathrm{pH}$ changes, which are typically proportional to the analyte concentration. Most prominent example for this concept are glucose biosensors to test the level of glucose in a droplet of blood, which are commercially very widespread. [2] In the field of biosensing research affinity-based biosensors, where the target analyte is captured by specific capture molecules immobilized at the sensor surface of a device, are also very commonly used. [3] These devices also show very good performance and they have been reported to be very sensitive. The sensing effect is caused by the surface binding of the molecules to their target counterparts, which is then read out by a specific transducer principle such as mass change, thermal change, optical signals or electrochemical effects.

However, this type of biosensor has two major drawbacks: Firstly it also shows signals when non-specific binding of competing molecules from the analyte solution to the sensor surface occurs. Secondly, the sensor surface can only offer a certain number of binding sites per unit area, which leads to a saturation of the sensing signal at elevated analyte concentration levels. This is then usually described by binding isotherms such as the Langmuir binding isotherm. [4] In contrast to the abovementioned, enzyme-based biosensor these label-free concepts, however, are much more robust in terms of shelf-life of the sensors.

In our biosensor concept we use arrays of topdown fabricated silicon nanowire field-effect transistors (SINW FETs) as transducers. These devices can be regarded as a modern version of the classical ion-sensitive field-effect transistor (ISFET) concept, which was firstly introduced by P. Bergveld in 1970. [5] In the last two decades this concept was miniaturized to the SiNW FET format pioneered by the group of C. M. Lieber. [6] Many publications followed 
up leading to a renaissance of the classical ISFET concepts from the 70's and 80's. Two fabrication protocols such as bottom-up [7] and top-down fabrication [8] are applied in the field of SiNW FETs. In many of the bioassays described with this type of devices, the affinitybased concept is used. Due to their ultimate miniaturization the SiNW FETs were reported to be much more sensitive than the classical ISFET sensors. Typically much smaller currents are flowing through the devices during detection, which also complicates the amplifier design for point of care systems but offers the possibility for a much smaller power consumption of the sensors.

With this gain in sensitivity combined with the general drawback of unspecific binding inherent to the affinity-based biosensor concepts, the researchers also buy in a higher influence of side-parameters in the bioassay, where $\mathrm{pH}$, ionic strength, and temperature changes in the solution are the most dominant ones. In this contribution we will present tests to these three side parameters with our SiNW FET platform and suggest a solution how to tackle these fundamental problems in biomedical point of care detection.

\section{Results and Discussion}

Biosensor devices need to be operated in liquid environments, since most of the time patient samples from blood serum, urine or sputum are tested. Such sensor concepts are applied to many different applications in the biomedical field. Usually the surfaces of the sensor inputs are decorated with capture biomolecules, which guarantee the specificity of the sensor to a respective type of analyte molecule. This is also the case for our SiNW FETs, which we operate in a classical ISFET configuration using a front gate contact to the liquid solution via an electrochemical $\mathrm{Ag} / \mathrm{AgCl}$ reference electrode and a back gate contact to the bulk silicon wafer (Fig. 1).

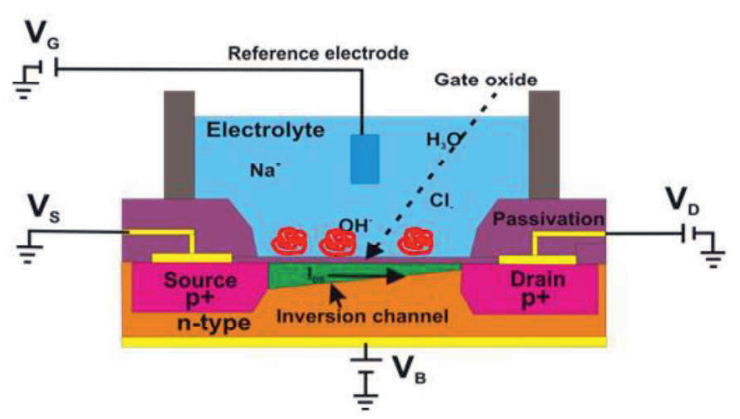

Fig. 1. Classical ISFET configuration for the operation of a sensor device: Typically a drain source voltage $V_{D S}$ and a gate-source voltage $V_{G S}$ are applied. In our case also the back gate contact can be set to a respective potential $V_{B S}$.
When analyte molecules bind to the sensor surface this event is then translated into a measurable signal.

In case of the SiNW FET sensors, the transistor devices are set into a working point by applying a voltage between gate and drain contacts $V_{D S}$, a voltage at the front gate contact of the electrolyte solution $V_{G S}$ and a back gate voltage $\mathrm{V}_{\mathrm{BS}}$. In such a working point a constant current in the range of $1 \mu \mathrm{A}$ and less is flowing through each nanowire. In case of even smaller nanowire diameters used by others currents in the nA regime are very typical. In some of the publications the researchers even used the subthreshold regime of the nanowire devices, with then much smaller currents.

The main transducer mechanism used for the lable-free electronic detection of biomolecules with ISFET devices is based on the change of the surface potential of the solid-liquid interface at the sensor gate input. This is leading to a shift in threshold voltage of the devices. It can either be measured from taking repetitive transfer characteristics while extracting the shift in threshold voltage $\mathrm{V}_{\mathrm{TH}}$ or a time-dependent readout can be applied. For this readout concept the devices are set into a specific working point by selecting a particular $\mathrm{V}_{\mathrm{DS}}$ and $V_{G S}$ combination and the change in current $I_{D S}$ is then followed by means of an amplifier.

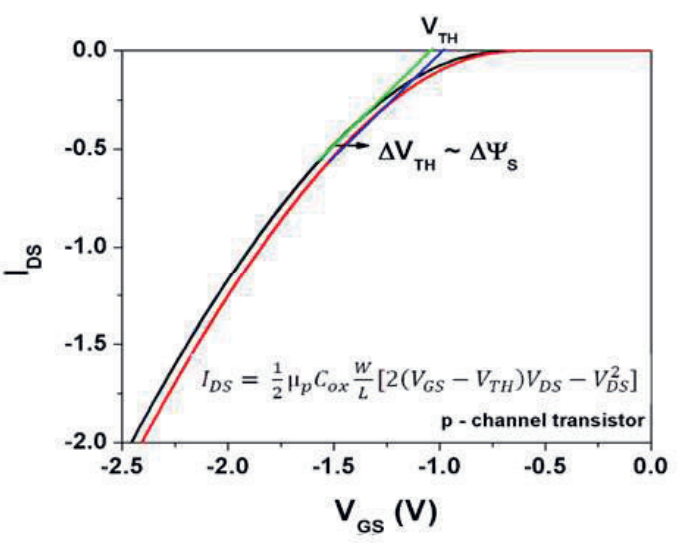

Fig. 2. Transfer characteristics of a p-type ISFET device. The typical currents $I_{D S}$ are in the range of 0.2-1.0 mA for our ISFET devices and a factor of 1000 smaller for the SiNW ISFETs $(0.2-1.0 \mu \mathrm{A})$. In both cases we operate the devices in the linear regime where the equation in the inset applies. A change of the surface potential at the sensor input $\Psi_{S}$ is changing the threshold voltage $V_{T H}$ of the device.

The change in surface potential upon binding of charged biomolecules to a sensor surface in an electrolyte solution can be described by the Poisson Boltzmann theory and many works were dedicated to develop and adjust this theory for ISFET devices. [9, 10] 
An alternative theory, where the biomolecule charges are not directly detected, but the cloud of ions surrounding them, was also described. [11] Both theories have in common that they explain the change in surface potential upon biomolecule binding and hence a change in the devices' threshold voltage $V_{T H}$. Recently we described that eventually two effects of change in $\mathrm{V}_{\mathrm{TH}}$ and recruiting of charge carriers into the wires might be combined explaining the higher sensitivity reported in the field for SINW FETs in comparison with classical, micro-meter sized ISFETs. [12]

The typical change in current through the nanowires at a fixed bias potential has in many work been used to display the wire conductance vs. time. [6, 7] It is well known for semiconductor devices, that the charge carrier density of the semiconductor material is largely influenced by temperature changes. This can also be observed when using these devices, especially when small wires with thin diameters and very high signal amplification is used.

From the classical ISFET approach it is also well-known that the primary response of such a device is towards changes in $\mathrm{pH}$ of the test solution. [5] In addition, especially when the passivation layer of the contact lines or even the gate dielectrics shows a leakage effect (in particular when only native $\mathrm{SiO}_{2}$ is used at the sensor surface), ionic strength changes in the liquid may also highly influence the recorded signals. Therefore the reactions of the sensors to these three main side parameters should always be checked prior usage for biomedical assays.

In recent years we alternatively applied an impedimetric readout approach for our ISFET and SiNW FET devices, which is based on a slightly different transducer mechanism. [13] Here the input impedance of the device is sensed which is linked to capacitance changes at the solid-liquid interface caused by capacitive screening and by immobilized charges. This can alternatively be utilized to detect biomolecules. [14] With this transducer mechanism also the attachment of individual cells to ISFET devices can be measured. [15] In this approach, however, the sensor response is not very sensitive to $\mathrm{pH}$ changes and temperature effects, but the ionic strength of the test solution plays a major role.

For both readout principles applying a differential readout between identical sensors in an array format is leading to the most reliable results. Most of the problems for unspecific binding of molecules can be cancelled out by this approach. Also $\mathrm{pH}$, temperature and ionic strength effects are usually suppressed and therefore we regard a differential approach as inevitable for a reliable readout of biosensors.

\section{Conclusion and Outlook}

There are several important demands for stable and reliable readout of biosensor devices especially when nano-scale device with small electronic carrier signals are used. A first prerequisite is the usage of a stable reference electrode such as a liquid-junction $\mathrm{Ag} / \mathrm{AgCl}$ electrode to apply the front gate voltage. Also a differential readout approach to compare a sensor element with an ideally inert reference element towards analyte binding should be applied. In addition it is advised to control and readout the above-mentioned side parameters temperature, $\mathrm{pH}$ and ionic strength of the test solution during detection. For a portable system the sensor integration and the combined sensor concept need to be optimized and the sensor outputs need to be evaluated in terms of crosssensitivity, feedback and hysteresis.

In our SiNW FET biosensor project we aim to combine the various sensor inputs such as potentiometric and impedance readout, temperature sensing, ionic strength sensing and $\mathrm{pH}$ sensing into a combined, multiparametric system approach. For portable applications the discrete amplifier components needs to be realized with low power consumption. Nano-scale sensor devices have a real strength in this respect since the power consumption can be minimized with such systems.

\section{Acknowledgements}

We like to acknowledge financial support by the EUROIMMUN Medizinische Labordiagnostika AG, Germany, and by the European Commission Program through the Marie Curie Initial Training Network PROSENSE (grant no. $317420,2012-2016)$. Various other funding projects by University of Applied Sciences Kaiserslautern (UASK) and several BMBF projects funded the past research works of $\mathrm{PhD}$ students in our team. They largely contributed to the development of the SiNW FET platform. In addition the technical staff of UASK is acknowledged for support.

\section{References}

[1] J. Wang, Electrochemical biosensors: Towards point-of-care cancer diagnostics, Biosensors and Bioelectronics 21, 1887-1892 (2006); doi: 10.1016/j.bios.2005.10.027

[2] J. Wang, Electrochemical Glucose Biosensors, Chemical Reviews 108, 814-825 (2008); doi: $10.1021 / \mathrm{cr068123a}$ 
[3] K.R. Rogers, Principles of affinity-based biosensors, Molecular Biotechnology 14, 109-129 (2000); doi:10.1385/MB:14:2:109

[4] J. S. Daniels, N. Pourmand, Label-Free Impedance Biosensors: Opportunities and Challenges, Electroanalysis. 19, 1239-1257 (2007); doi: 10.1002/elan.200603855

[5] P. Bergveld, Development of an Ion-Sensitive Solid-State Device for Neurophysiological Measurements. IEEE Transactions on Biomedical Engineering 17, 70-71 (1970); doi: 10.1109/TBME.1970.4502688

[6] Y. Cui, Q. Wei, H. Park, C. M. Lieber, Nanowire nanosensors for highly sensitive and selective detection of biological and chemical species, Science 293, 1289-1292 (2001); doi: 10.1126/science. 1062711

[7] F. Patolsky, G. Zheng, C. M. Lieber, Fabrication of silicon nanowire devices for ultrasensitive, label-free, real-time detection of biological and chemical species, Nature Protocols 1, 1711-1724 (2006); doi:10.1038/nprot.2006.227

[8] Z. Li, Y. Chen, X. Li, T. I. Kamins, K. Nauka, R. S. Williams, Sequence-Specific Label-Free DNA Sensors Based on Silicon Nanowires, Nano Letters 4, 245-247 (2004); doi: 10.1021/nl034958

[9] D. Landheer, G. Aers, W. R. McKinnon, Model for the field effect from layers of biological macromolecules on the gates of metal-oxidesemiconductor transistors, Journal of Applied Physics 98, 044701 (2005); doi: 10.1063/1.2008354

[10] M. W. Shinwari, M. J. Deen, D. Landheer, Study of the electrolyte-insulator-semiconductor fieldeffect transistor (EISFET) with applications in biosensor design, Microelectronics Reliability 47, 2025-2057 (2007); doi: 10.1016/j.microrel.2006.10.003

[11] A. Poghossian*, A. Cherstvy, S. Ingebrandt, A. Offenhäusser, and M.J. Schöning, Possibilities and limitations of label-free detection of DNA hybridization with field-effect-based devices, Sensors and Actuators B - Chemical 111-112, 470-480 (2005); doi: 10.1016/j.snb.2005.03.083

[12] M. Schwartz, T. C. Nguyen, X. T. Vu, M. Weil, J. Wilhelm, P. Wagner, R. Thoelen, S. Ingebrandt, DNA detection with top-down fabricated silicon nanowire transistor arrays in linear operation regime, Physica Status Solidi A 213, 1510-1519 (2016); doi: 10.1002/pssa.201532919

[13] S. Ingebrandt*, Y. Han, F. Nakamura, A. Poghossian, M. J. Schöning, A. Offenhäusser, Label-free detection of single nucleotide polymorphisms utilizing the differential transfer function of field-effect transistors, Biosensors and Bioelectronics 22, 2834-2840 (2007); doi: 10.1016/j.bios.2006.11.019

[14] T. C. Nguyen, X. T. Vu, M. Freyler, S. Ingebrandt, PSPICE model for silicon nanowire based biosensors in impedimetric measurement mode,
Physica Status Solidi A 210, 870-876 (2013); doi: 10.1002/pssa.201200919

[15] A. Susloparova, D. Koppenhöfer, J. K. Y. Law, X. T. Vu, S. Ingebrandt, Electrical Cell-substrate Impedance Sensing with field-effect transistors is able to unravel cellular adhesion and detachment processes on a single cell level, Lab on a Chip 15, 668-679 (2015); doi: 10.1039/C4LC00593G 